\title{
KR-12-a6 promotes the osteogenic differentiation of human bone marrow mesenchymal stem cells via BMP/SMAD signaling
}

\author{
LANQING FU ${ }^{1 *}$, PEICHENG JIN ${ }^{2 *}$, YAJUN HU ${ }^{3}$, HOUGEN LU ${ }^{1}$ and LINLIN SU ${ }^{4}$ \\ ${ }^{1}$ Department of Orthopedics, Jingzhou Central Hospital, Tongji Medical College of Huazhong University of \\ Science and Technology, Jingzhou, Hubei 434020; ${ }^{2}$ Department of Orthopedics, Xiangyang No. 1 People's Hospital, \\ Affiliated Hospital of Hubei University of Medicine, Xiangyang, Hubei $441000 ;{ }^{3}$ Department of Gynecology and Obstetrics, \\ Jingzhou Central Hospital, Tongji Medical College of Huazhong University of Science and Technology, \\ Jingzhou, Hubei 434020; ${ }^{4}$ Department of Burns and Cutaneous Surgery, Xijing Hospital, \\ The Fourth Military Medical University, Xi'an, Shaanxi 710032, P.R. China
}

Received March 4, 2019; Accepted July 5, 2019

DOI: $10.3892 / \mathrm{mmr} .2019 .10843$

\begin{abstract}
Considering the increased resistance to antibiotics in the clinic and the ideal antibacterial properties of KR-12, the effects of KR-12-a6, an important analogue of KR-12, on the osteogenic differentiation of human bone marrow mesenchymal stem cells (hBMSCs) were investigated. Osteogenic differentiation-associated experiments were conducted in hBMSCs, and KR-12-a6 was used as an additional stimulating factor during osteogenic induction. Quantitative analysis of alkaline phosphatase (ALP) and alizarin red staining, and reverse transcription-quantitative PCR analysis of the expression of osteogenesis-associated genes were performed to determine the effects of KR-12-a6 on the osteogenic differentiation of hBMSCs. LDN-212854 was selected to selectively suppress BMP/SMAD signaling. Western blotting was performed to investigate the underlying mechanisms. The intensity of ALP and alizarin red staining gradually increased with increasing KR-12-a6 concentrations. KR-12-a6 induced the strongest staining at $40 \mu \mathrm{g} / \mathrm{ml}$, whereas $60 \mu \mathrm{g} / \mathrm{ml}$ and $80 \mu \mathrm{g} / \mathrm{ml}$ concentrations did not further increase the intensity
\end{abstract}

Correspondence to: $\mathrm{Dr}$ Linlin $\mathrm{Su}$, Department of Burns and Cutaneous Surgery, Xijing Hospital, The Fourth Military Medical University, 127 Changle West Road, Xincheng, Xi'an, Shaanxi 710032, P.R. China

E-mail: linlinsu@fmmu.edu.cn

Dr Hougen Lu, Department of Orthopedics, Jingzhou Central Hospital, Tongji Medical College of Huazhong University of Science and Technology, 60 Jingzhong Road, Jingzhou, Jingzhou, Hubei 434020, P.R. China

E-mail: luhg_jzch@163.com

*Contributed equally

Key words: KR-12-a6, antimicrobial peptide, osteogenic differentiation, human bone marrow mesenchymal stem cells, bone morphogenic protein/SMAD signaling of staining. The mRNA expression levels of RUNX2 and ALP increased in a dose-dependent manner as early as 3 days post-KR-12-a6 treatment. The mRNA expression of COL1A1, $B S P$ and $B M P 2$ exhibited significant upregulation from day 7 post-KR-12-a6 treatment. In contrast, the mRNA levels of $O S X, O C N$ and $O P N$ were enhanced dramatically at day 14 following KR-12-a6 stimulation. Additionally, KR-12-a6 significantly promoted the phosphorylation of Smad1/5. Furthermore, LDN-212854 suppressed the activation of Smad1/5 and inhibited the upregulation of several osteogenic differentiation-associated genes in KR-12-a6-treated hBMSCs. KR-12-a6 promoted the osteogenic differentiation of hBMSCs via $\mathrm{BMP} / \mathrm{SMAD}$ signaling.

\section{Introduction}

As an important part of the immune system, antimicrobial peptides (AMPs) can physically destroy microbial membranes and induce their cleavage, indicating their potential as substitutes for traditional antibiotics (1). Following the identification of LL-37, the only natural human cathelicidin (an AMP subfamily), in 1995 (2,3), its presence has been detected in various types of cells and epithelia, including intestinal epithelial cells (4), mast cells (5), monocytes (6) and lymphocytes (7). Natural human AMPs are the first line of defense against local infection, serving an important role in the invasion of systemic pathogens into local areas or local wound infections $(8,9)$. As the peptide chain of LL-37 is too long and difficult to synthesize, it is not a routine therapeutic drug for bacterial infections or other inflammatory diseases in the clinic; however, the cost of producing short-chain AMPs is relatively low. Furthermore, the removal of hydrophobic amino acids from the N-terminus of natural LL-37 can reduce its toxicity to eukaryotic cells, as well as reduce its interactions with human plasma proteins $(10,11)$. Thus, short-chain AMPs have attracted the attention of researchers. Compared with natural LL-37, KR-20 and KS-30, two derivatives of LL-37, exhibit improved antimicrobial properties $(12,13)$. KR-12 is the shortest derivative of LL-37 with antimicrobial activity $(10,11)$. Compared with LL-37 and its other derivatives, the cost of KR-12 synthesis 
is lower, and its cytotoxicity is reduced; for example, LL-37, but not KR-12, induces hemolytic effectsin human erythrocytes (14). As an analogue of KR-12 (amino acid sequence: KRIVQRIKDFLR-NH ${ }_{2}$ ), KR-12-a6 (amino acid sequence: LRIVKLILKWLR-NH ${ }_{2}$ ) has similar antibacterial properties and biocompatibility (15); also, as a highest hydrophobic analogue of KR-12, KR-12-a6 displays greater inhibition of lipopolysaccharide (LPS)-stimulated tumor necrosis factor- $\alpha$ production and higher LPS-binding activity than KR-12 (15). Therefore, KR-12-a6 may be a potential therapeutic drug for the treatment of clinical infectious diseases, including osteomyelitis.

Osteomyelitis is a common infectious disease that can cause serious symptoms locally or systemically, such as severe pain, high fever, swelling and tenderness around the infection site, posing a great challenge to clinical treatment (16). In addition to the surgical removal of infected bone tissues, local or systemic application of antibiotics is the most common treatment (17); however, with the increasing use of antibiotics to treat bone infections in the clinic, bacterial resistance to antibiotics has gradually developed (18). In addition, osteolysis caused by infection cannot be treated with traditional antibiotics. Gentamicin (19) and vancomycin (20), two commonly used antibiotics in the treatment of bone-related infections, have been shown to inhibit the viability of osteoblasts and reduce their numbers. Recently, certain small polypeptides have been reported to promote bone integration while controlling infection (21). Therefore, drugs with favorable antibacterial properties and pro-osseointegration abilities are of great importance for the treatment of clinical bone infections.

Human bone marrow mesenchymal stem cells (hBMSCs) play a key role in the process of bone regeneration (22-24). hBMSCs can differentiate into osteoblasts during bone formation, and are the main source of bone progenitor cells (25). When local infections are controlled, hBMSCs are activated and differentiate into osteoblasts to repair the local osteolysis (26). However, when hBMSCs fail to completely repair the local bone defect caused by infection, local osteoporosis and even pathological fracture can result (27). At present, drugs that inhibit osteoclast activity and bone resorption, such as vitamin $\mathrm{D}$ analogues, calcitonin and estrogen, are commonly used in the treatment of osteoporosis (28). However, these drugs do not promote osteogenic differentiation or exhibit antibacterial properties. Considering the increased resistance to antibiotics in the clinic and the ideal antibacterial properties of AMPs, it was proposed that AMPs may exhibit positive therapeutic effects for the treatment of osteomyelitis (29). Therefore, the present study examined whether its analogue KR-12-a6 could also promote the osteogenic differentiation of hBMSCs and investigated the underlying mechanisms.

\section{Materials and methods}

Isolation and culture of hBMSCs. Bone marrow was obtained from the iliac crest marrow aspirates of 3 healthy donors (one 34-year old male, two females at 19 and 38-year's old) undergoing iliac crest bone transplantation at Jingzhou Central Hospital, Tongji Medical College of Huazhong University of Science and Technology (Jingzhou, China) between January and July 2018. The procedure was performed following approval from the Ethics Committee of Huazhong University of Science and Technology and after obtaining donors' informed consent. The collected bone marrow was treated with heparin anticoagulant and then diluted in $\alpha$-minimal essential medium ( $\alpha$-MEM; Gibco; Thermo Fisher Scientific, Inc.) (30). hBMSCs were then isolated by density gradient centrifugation ( $400 \mathrm{x} \mathrm{g}$ for $20 \mathrm{~min}$ at room temperature) with Ficoll-Paque (GE Healthcare) and plastic adherence, purified by discarding suspended cells through exchanging medium, and then grown in $\alpha$-MEM supplemented with $20 \%$ fetal bovine serum (Atlanta Biologicals; Bio-Techne Corporation), $100 \mathrm{U} / \mathrm{ml}$ penicillin (Invitrogen; Thermo Fisher Scientific, Inc.), $100 \mu \mathrm{g} / \mathrm{ml}$ streptomycin (Invitrogen; Thermo Fisher Scientific, Inc.), and $2 \mathrm{mM}$ L-glutamine (Invitrogen; Thermo Fisher Scientific, Inc.). Cells were then cultured at $37^{\circ} \mathrm{C}$ with $5 \%$ humidified $\mathrm{CO}_{2}$. The hBMSCs were passaged and maintained at low density. Adherent cells reaching $80 \%$ confluence were harvested by using $0.25 \%$ trypsin at $37^{\circ} \mathrm{C}$ for $3 \mathrm{~min}$. Then, $1 \times 10^{5}$ cells were replated, and the remainder was used for the analysis of gene expression.

Osteoblast differentiation. hBMSCs were plated in 6-well plates in standard growth medium. At $80 \%$ confluence, the medium was replaced with osteogenic medium consisting of $\alpha$-MEM $+10 \%$ fetal calf serum (Gibco; Thermo Fisher Scientific, Inc.) and $1 \%$ penicillin/streptomycin supplemented with $10 \mathrm{mM} \beta$-glycerophosphate (Calbiochem; Merck KGaA), $50 \mu \mathrm{g} / \mathrm{ml} \mathrm{L}$-ascorbic acid (Wako Chemicals $\mathrm{GmbH}$ ), $10 \mathrm{nM}$ dexamethasone (Sigma-Aldrich; Merck KGaA), and $10 \mathrm{nM}$ calcitriol (Sigma-Aldrich; Merck KGaA). The medium was replaced every 3 days. The control cells were grown in standard growth medium. Cell pellets were harvested for RNA isolation at different time points following induction.

Alkaline phosphatase (ALP) staining and quantitative analysis. hBMSCs were cultured in 24-well plates at $2 \times 10^{4}$ cells $/ \mathrm{cm}^{2}$. At $80 \%$ confluence, cells were induced to differentiate into osteoblasts using StemPro ${ }^{\mathrm{TM}}$ Osteogenesis Differentiation kit (Gibco; Thermo Fisher Scientific, Inc.) supplemented with KR-12-a6 (synthesized and purified by GL Biochem (Shanghai) Ltd.) at $0,20,30,40,60$, or $80 \mu \mathrm{g} / \mathrm{ml}$ at $37^{\circ} \mathrm{C}$ for 7 days. For ALP staining, hBMSCs in each group were washed twice with PBS at the end of the 7-day KR-12-a6-treatment period, fixed with $4 \%$ paraformaldehyde for $30 \mathrm{~s}$, and then stained using the ALP staining kit (Maokang Biotechnology, Shanghai) according to the manufacturer's instructions. Images were acquired by an inverted phase contrast microscope (CKX41, Olympus) under x100 magnification. At least five fields per sample were randomly selected and observed. To quantitatively analyze ALP activity, hBMSCs after the 7-day KR-12-a6-treatment period were washed twice with PBS and lysed with Triton X-100 (1\%) for $15 \mathrm{~min}$. Then, the activity was measured by Alkaline Phosphatase Assay kit (Beyotime Institute of Biotechnology) according to the manufacturer's instructions. The final estimation was based on the absorbance at $405 \mathrm{~nm}$ measured by a spectrophotometer.

Alizarin red staining and quantitative analysis. Assessment of ex vivo mineralization was performed by employing alizarin red staining. hBMSCs at the density of $5 \times 10^{4}$ cells/well 
Table I. Primer sequences for reverse transcription-quantitative PCR.

\begin{tabular}{lll}
\hline Gene & \multicolumn{1}{c}{ Forward primer } & \multicolumn{1}{c}{ Reverse primer } \\
\hline RUNX2 & 5'-GCCACCACTCACTACCACACCTA-3' & 5'-TCCTGACGAAGTGCCATAGTAGAGATAT-3' \\
ALP & 5'-GGACCATTCCCACGTCTTCAC-3' & 5'-CCTTGTAGCCAGGCCCATTG-3' \\
$C O L 1 A 1$ & 5'-TGGGAGGAAGCAAAAGACTC-3' & 5'-GGGTCATTTCCACATGCTTT-3' \\
$B S P$ & 5'-TGCCTTGAGCCTGCTTCC-3' & 5'-GCAAAATTAAAGCAGTCTTCATTTTG-3' \\
$B M P 2$ & 5'-AACACTGTGCGCAGCTTCC-3' & 5'-CTCCGGGTTGTTTTCCCAC-3' \\
OSX & 5'-CCCCACCTCTTGCAACCA-3' & 5'-CCCCACCTATTGCAACCA-3' \\
OPN & 5'-GCCGACCAAGGAAAACTCACT-3' & 5'-GGCACAGGTGATGCCTAGGA-3' \\
$\beta$ - actin & 5'-CCCAGGCGCTACCTGTATCAA-3' & 5'-GGTCAGCCAACTCGTCACAGTC-3' \\
\hline
\end{tabular}

$A L P$, alkaline phosphatase; $B M P$, bone morphogenic protein; $B S P$, bone sialoprotein; $C O L 1 A 1$, type 1 collagen $\alpha 1$ chain; $O C N$, osteocalcin; $O P N$, osteopontin; $O S X$, osterix; $R U N X 2$, runt-related transcription factor 2.

in 6-well plates underwent osteoblast differentiation in medium supplemented with KR-12-a6 at 0, 20, 30, 40, 60, or $80 \mu \mathrm{g} / \mathrm{ml}$ at $37^{\circ} \mathrm{C}$ for 21 days. Then cells were washed in PBS, fixed in $70 \%$ ethanol at $-20^{\circ} \mathrm{C}$ for $1 \mathrm{~h}$, and rinsed in $\mathrm{dH}_{2} \mathrm{O}$. The cultures were stained with $40 \mathrm{mM}$ alizarin red (Sigma-Aldrich; Merck KGaA) at $\mathrm{pH} 4.2$ for $10 \mathrm{~min}$ at room temperature with rotation. Cells were then rinsed twice with $\mathrm{dH}_{2} \mathrm{O}$, followed by washing three times with PBS to reduce nonspecific staining. Images were acquired by an inverted phase contrast microscope (CKX41, Olympus Corporation) under x100 magnification. At least five fields per sample were randomly selected and observed. For further quantitative analysis, a $10 \%$ chlorinated 16 alkyl pyridine solution of sodium phosphate ( $\mathrm{pH} 7.0)$ was added into each sample to dissolve the dye, and the absorbance was measured at $620 \mathrm{~nm}$ by a spectrophotometer.

$R N A$ isolation and reverse transcription-quantitative PCR $(R T-q P C R)$. hBMSCs at the density of $5 \times 10^{4}$ cells/well in 12-well plates were treated with KR-12-a6 at 0, 20, 30, and $40 \mu \mathrm{g} / \mathrm{ml}$ and then cultured at $37^{\circ} \mathrm{C}$ for 3,7 , and 14 days. On day 3,7 , and 14 post-KR-12-a6 treatment, hBMSCs were harvested in Buffer RLT using an RNeasy Mini kit (Qiagen, Inc.). Total RNA was prepared according to standard protocols. Complementary DNAs (cDNAs) were synthesized from $0.5 \mu \mathrm{g}$ total RNA using a QuantiTect Reverse Transcription kit (Qiagen, Inc.) according to the manufacturer's protocols. qPCR was conducted using a QuantiTect SYBR Green PCR kit (Qiagen, Inc.) and an ABI PRISM 7900HT SDS instrument (Applied Biosystems; Thermo Fisher Scientific, Inc.). qPCR was performed as follows: 2 min activation at $95^{\circ} \mathrm{C}$, then 40 cycles of $15 \mathrm{sec}$ denaturation at $95^{\circ} \mathrm{C}$ and $15 \mathrm{sec}$ annealing at $62^{\circ} \mathrm{C}$, and a final $20-\mathrm{sec}$ extension at $68^{\circ} \mathrm{C}$. All reactions were in a final volume of $20 \mu \mathrm{l}$ consisting of $2 \mathrm{X}$ master mix $(10 \mu \mathrm{l})$, forward and reverse primers $(2 \mu \mathrm{l})$, cDNA, and RNase $\mathrm{H}_{2} \mathrm{O}$, and each sample was run in triplicate. The specificity of PCR products was confirmed at the end of each run via melting curve analysis. All signals were normalized to $\beta$-actin, and the quantification cycle was determined. RT-qPCR was quantified by $2^{-\Delta \Delta C q}$ method (31). Oligonucleotide primers used for RT-qPCR are presented in Table I. $\beta$-actin was used as the housekeeping gene to normalize gene expression levels.

Western blot analysis. hBMSCs at the density of $5 \times 10^{4}$ cells/well in 12-well plates were treated with KR-12-a6 at $0,20,30$, and $40 \mu \mathrm{g} / \mathrm{ml}$ and then cultured at $37^{\circ} \mathrm{C}$ for 7 days. On day 7 post-KR-12-a6 treatment, hBMSCs were harvested and lysed in immunoprecipitation lysis buffer (10 mM Tris, $0.15 \mathrm{M} \mathrm{NaCl}, 1 \% \mathrm{NP}-40$, and $10 \%$ glycerol, $\mathrm{pH} 7.4$, at $22^{\circ} \mathrm{C}$ ) containing protease and phosphatase inhibitor cocktails (Sigma-Aldrich; Merck KGaA). Cell lysates were then centrifuged at $15,000 \mathrm{x}$ g for $45 \mathrm{~min}$ at $4^{\circ} \mathrm{C}$. The supernatants were collected, and the protein concentration was determined by BCA assay with a Bio-Rad Model 680 Plate Reader. Then, $40 \mu \mathrm{g} / \mathrm{sample}$ of protein was separated via $7.5 \%$ SDS-PAGE and transferred onto nitrocellulose membranes. The membranes were blocked with $5 \%$ non-fat milk for $1 \mathrm{~h}$ at $4^{\circ} \mathrm{C}$, and then incubated with primary antibodies targeting phosphorylated (p)-Smad1/5 (cat. no. 9516S, Cell Signaling Technology, 1:500 dilution), Smad1/5 (cat. no. sc-6201, Santa Cruz Biotechnology, 1:200 dilution), or $\beta$-actin (cat. no. sc-1616, Santa Cruz Biotechnology, 1:200 dilution) overnight at $4{ }^{\circ} \mathrm{C}$. The following day, membranes were washed and incubated with bovine anti-rabbit IgG-HRP secondary antibody (cat. no. sc-2370, Santa Cruz Biotechnology, 1:1,000 dilution) or bovine anti-goat IgG-HRP secondary antibody (cat. no. sc-2350, Santa Cruz Biotechnology, 1:1,000 dilution) for $1 \mathrm{~h}$ at room temperature, and immunoreactive bands were detected and visualized by ECL reagent (EMD Millipore) and a FluorChem FC Imaging system (Alpha Innotech). The protein expression was then quantified by AlphaEase FC StandAlone software (version 6.0.0.14, Alpha Innotech).

$L D N-212854$ treatment of hBMSCs. hBMSCs at the density of $5 \times 10^{4}$ cells/well in 12-well plates were pre-treated with $10 \mu \mathrm{M}$ LDN-212854 (Selleck Chemicals, Shanghai) or DMSO (vehicle control) at $37^{\circ} \mathrm{C}$ for $14 \mathrm{~h}$, then washed 3 times with plain culture medium. hBMSCs continued to be cultured in medium containing $1 \mu \mathrm{M}$ LDN-212854 alone or $1 \mu \mathrm{M}$ LDN-212854 in combination with $40 \mu \mathrm{g} / \mathrm{ml} \mathrm{KR-12-a6}$ at $37^{\circ} \mathrm{C}$ 
A
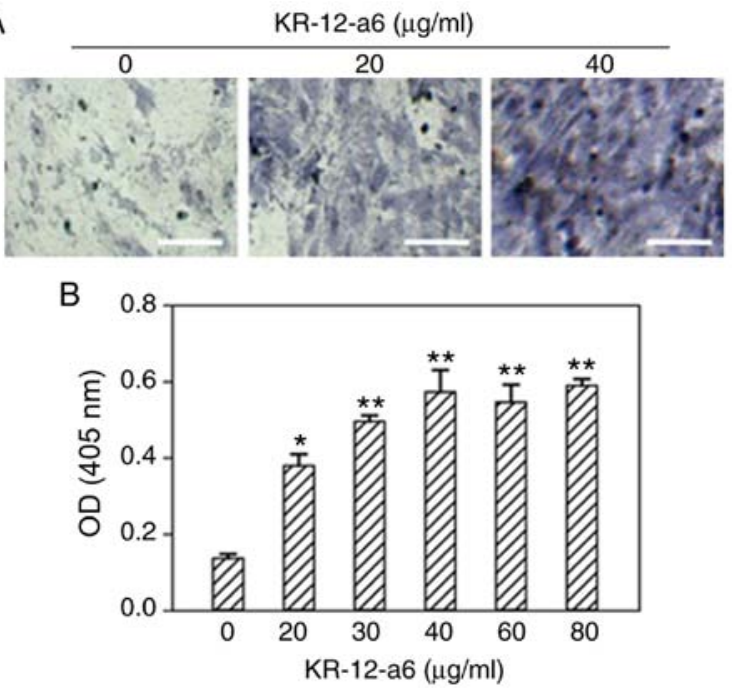

Figure 1. Osteogenic ALP staining of hBMSCs and quantitative analysis following KR-12-a6 treatment. ALP staining was performed following KR-12-a6 stimulation at different concentrations $(0,20,30,40,60$, and $80 \mu \mathrm{g} / \mathrm{ml}$ ) in hBMSCs. (A) Representative images following stimulation with 0,20 , and $40 \mu \mathrm{g} / \mathrm{ml} \mathrm{KR}-12-\mathrm{a} 6$. (B) Optical density of staining at $405 \mathrm{~nm}$. Data are presented asthe mean $\pm \mathrm{SD}(\mathrm{n}=4) .{ }^{*} \mathrm{P}<0.05,{ }^{* *} \mathrm{P}<0.01$ vs. KR-12-a6 at $0 \mu \mathrm{g} / \mathrm{ml}$. Scale bar $=100 \mu \mathrm{m}$. ALP, alkaline phosphate; OD, optical density; hBMSC, human bone marrow mesenchymal stem cell.

for 7 days. After washing for times with PBS, hBMSCs were harvested and subjected to western blot analysis.

Statistical analysis. Each experiment was repeated at least three times, data are expressed as the mean \pm standard deviation. One-way analysis of variance (ANOVA) followed by Tukey's post hoc test was used to determine statistical significance. All statistical analyses were performed using SPSS 19.0 (IBM Corp.). $\mathrm{P}<0.05$ was considered to indicate a statistically significant difference.

\section{Results}

Intensity of ALP and alizarin red staining increases with elevated concentrations of KR-12-a6 in hBMSCs. To determine the effects of KR-12-a6 on the osteogenic differentiation of hBMSCs, ALP staining and alizarin red staining were performed. With increasing concentrations of KR-12-a6, the intensity of ALP (Fig. 1) and alizarin red staining (Fig. 2) also increased. KR-12-a6 at $40 \mu \mathrm{g} / \mathrm{ml}$ exhibited the strongest effects on both ALP (Fig. 1) and alizarin red staining (Fig. 2), whereas KR-12-a6 at 60 and $80 \mu \mathrm{g} / \mathrm{ml}$ did not notably increase the staining intensity compared with KR-12-a6 at $40 \mu \mathrm{g} / \mathrm{ml}$.

mRNA expression of osteoblastic differentiation-associated genes increases after KR-12-a6 stimulation at different time pointsin hBMSCs. The mRNA expression levels of osteoblastic differentiation-associated genes, including RUNX2 (encoding runt-related transcription factor 2; Fig. 3A), $A L P$ (Fig. 3B), COL1A1 (encoding type 1 collagen alpha 1 chain; Fig. $3 \mathrm{C}$ ), $B S P$ (encoding bone sialoprotein; Fig. 3D), BMP2 (encoding bone morphogenic protein 2; Fig. 3E), OSX (encoding osterix; Fig. 3F), OCN (encoding osteocalcin; Fig. 3G) and $O P N$ (encoding osteopontin; Fig. $3 \mathrm{H}$ ), were determined via

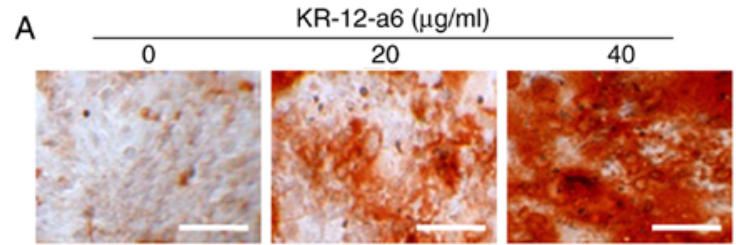

B

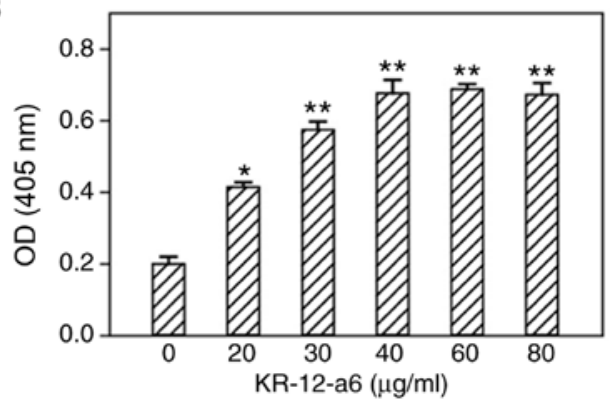

Figure 2. Osteogenic alizarin red staining of hBMSCs and quantitative analysis following KR-12-a6 treatment. Alizarin red staining was performed following KR-12-a6 stimulation at different concentrations $(0,20,30,40,60$, and $80 \mu \mathrm{g} / \mathrm{ml}$ ) in hBMSCs. (A) Representative images following stimulation with 0,20 , and $40 \mu \mathrm{g} / \mathrm{ml} \mathrm{KR-12-a6.} \mathrm{(B)} \mathrm{Optical} \mathrm{density} \mathrm{of} \mathrm{staining} \mathrm{at} 620 \mathrm{~nm}$. Data are presented as the mean $\pm \mathrm{SD}(\mathrm{n}=4) .{ }^{*} \mathrm{P}<0.05,{ }^{* *} \mathrm{P}<0.01$ vs. KR-12-a6 at $0 \mu \mathrm{g} / \mathrm{ml}$. Scale bar $=100 \mu \mathrm{m}$. OD, optical density; hBMSC, human bone marrow mesenchymal stem cell.

RT-qPCR analysis following treatment of hBMSCs with KR-12-a6 for 3, 7 or 14 days. The mRNA levels of RUNX2 and $A L P$ increased in a dose-dependent manner as early as 3 days post-KR-12-a6 treatment. The mRNA expression of COL1A1, BSP and BMP2 was significantly upregulated from day 7 post-KR-12-a6 treatment compared with the control. In contrast, the mRNA levels of $O S X, O C N$ and $O P N$ were only significantly upregulated at day 14 following KR-12-a6 stimulation.

BMP/SMAD signaling is activated during KR-12-a6-induced hBMSC osteogenic differentiation. As a significant elevation of $B M P 2$ mRNA was observed in Fig. 3E, it was next investigated as to whether BMP/SMAD signaling was involved in KR-12-a6-induced hBMSC osteogenic differentiation. The activation of SMAD signaling was examined via western blotting following KR-12-a6-induced hBMSC osteogenesis. The results showed that KR-12-a6 promoted the phosphorylation of Smad1/5 in a dose-dependent manner following 7 days of KR-12-a6 treatment (Fig. 4A and B) and exhibited the maximum activation at $40 \mu \mathrm{g} / \mathrm{ml}$. These results suggested that KR-12-a6 activated BMP/SMAD signaling in a dose-dependent manner.

Inhibition of $B M P / S M A D$ signaling suppresses KR-12-a6-induced osteogenic differentiation of hBMSCs. To further elucidate the role of BMP/SMAD signaling in osteoblast differentiation, LDN-212854, a novel BMP inhibitor that exhibits greater selectivity for BMP compared with the TGF- $\beta$ type I receptors, was used to suppress BMP/SMAD signaling. Western blotting was performed to observe the changes of several Smad proteins after 7 days of KR-12-a6 treatment with or without LDN-212854 (Fig. 5). The results showed that KR-12-a6 at $40 \mu \mathrm{g} / \mathrm{ml}$ significantly promoted the phosphorylation of Smad1/5 in hBMSCs, which was consistent with the 

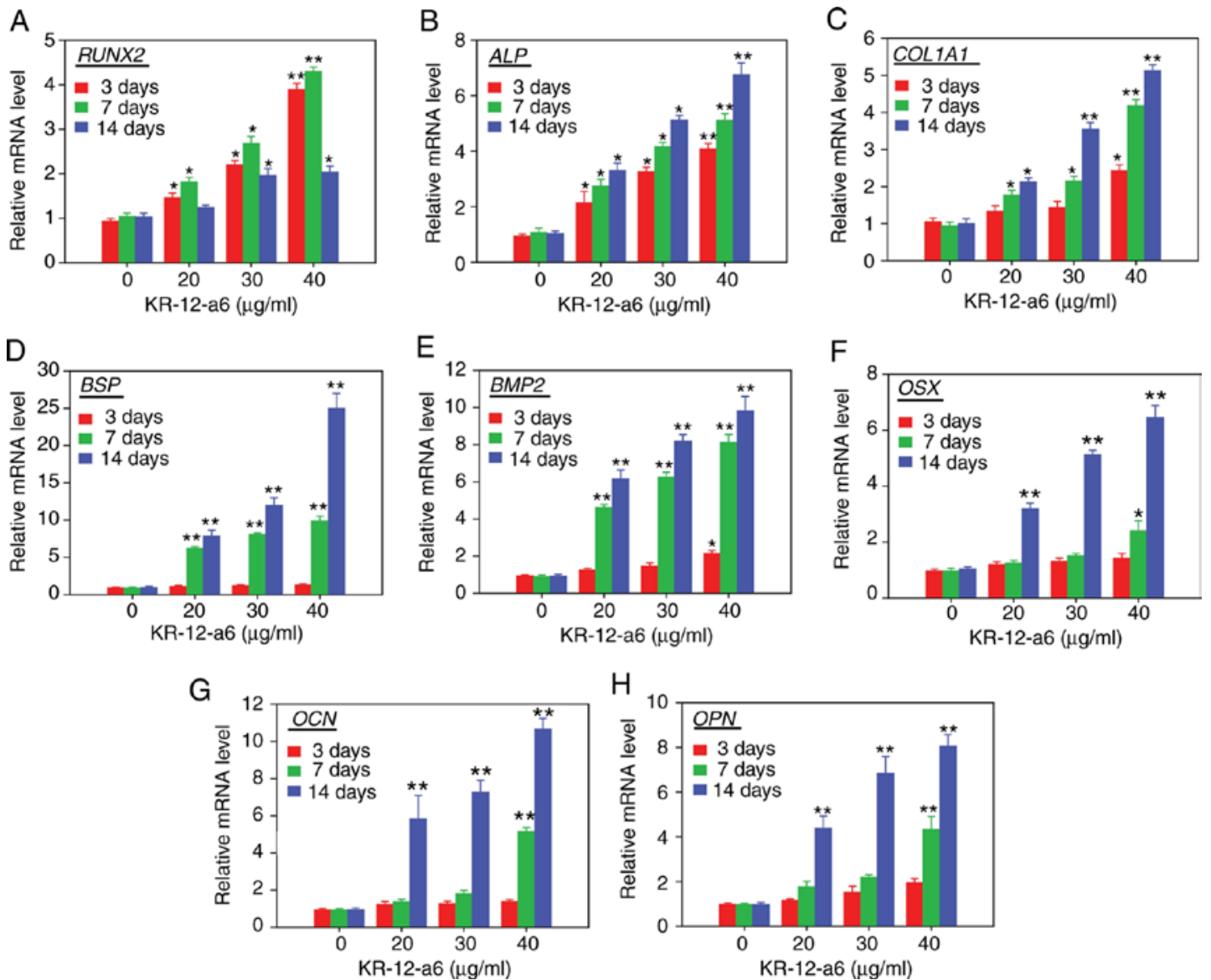

Figure 3. Effects of KR-12-a6 on the mRNA expression of osteogenic differentiation markers. Human bone marrow mesenchymal stem cells were treated with KR-12-a6 at concentrations of 0, 20, 30 or $40 \mu \mathrm{g} / \mathrm{ml}$, and the mRNA levels of (A) $R U N X 2$, (B) $A L P$, (C) COL1A1, (D) BSP, (E) BMP2, (F) $O S X$, (G) $O C N$ and (H) $O P N$ were determined via reverse transcription-quantitative PCR on days 3, 7 and 14 post-KR-12-a6 treatment. Data are presented as the mean $\pm \mathrm{SD}(\mathrm{n}=4)$. ${ }^{*} \mathrm{P}<0.05,{ }^{* *} \mathrm{P}<0.01$ vs. KR-12-a6 at $0 \mu \mathrm{g} / \mathrm{ml}$. $A L P$, alkaline phosphatase; $B M P$, bone morphogenic protein; $B S P$, bone sialoprotein; COL1A1, type 1 collagen $\alpha 1$ chain; $O C N$, osteocalcin; $O P N$, osteopontin; $O S X$, osterix; $R U N X 2$, runt-related transcription factor 2.

A

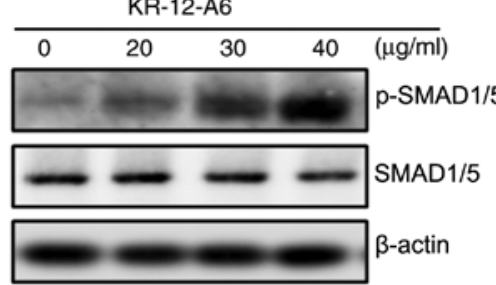

B

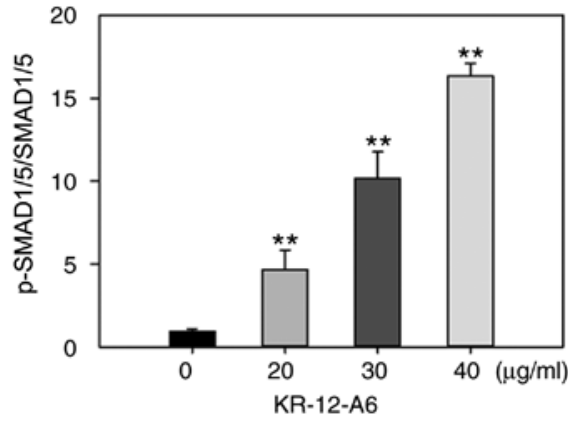

Figure 4. Effects of KR-12-a6 on the activation of BMP/SMAD signaling during the osteogenic differentiation of human bone marrow mesenchymal stem cells. (A) Western blotting was performed to determine the protein expression of $\mathrm{p}$-Smad1/5 and Smad1/5 after 7 days of KR-12-a 6 treatment at different concentrations $(0,20,30$ and $40 \mu \mathrm{g} / \mathrm{ml})$. $\beta$-actin served as the loading control. (B) Quantitative analysis of Smad1/5 phosphorylation. Data are presented as the mean $\pm \mathrm{SD}(\mathrm{n}=4) .{ }^{* *} \mathrm{P}<0.01$ vs. KR-12-a6 at $0 \mu \mathrm{g} / \mathrm{ml}$. p, phosphorylated.
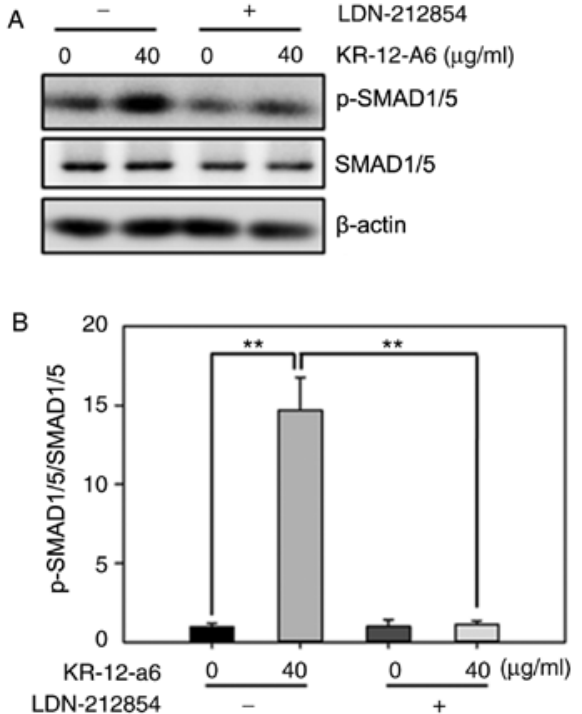

Figure 5. Effects of LDN-212854 on the activation of BMP/SMAD signaling in KR-12-a6-treated hBMSCs. hBMSCs were divided into four treatment groups as follows: Control, KR-12-a6, LDN-212854, and KR-12-a6+LDN-212854. (A) Western blot analysis of the protein expression of $\mathrm{p}$-Smad1/5 and Smad1/5 in the different treatment groups. $\beta$-actin served as the loading control. (B) Quantitative analysis of Smad1/5 phosphorylation. Data are presented as the mean $\pm \mathrm{SD}(\mathrm{n}=4) .{ }^{* *} \mathrm{P}<0.01$ vs. KR-12-a6 at $40 \mu \mathrm{g} / \mathrm{ml}$ without LDN-212854. hBMSC, human bone marrow mesenchymal stem cell; p, phosphorylated. 

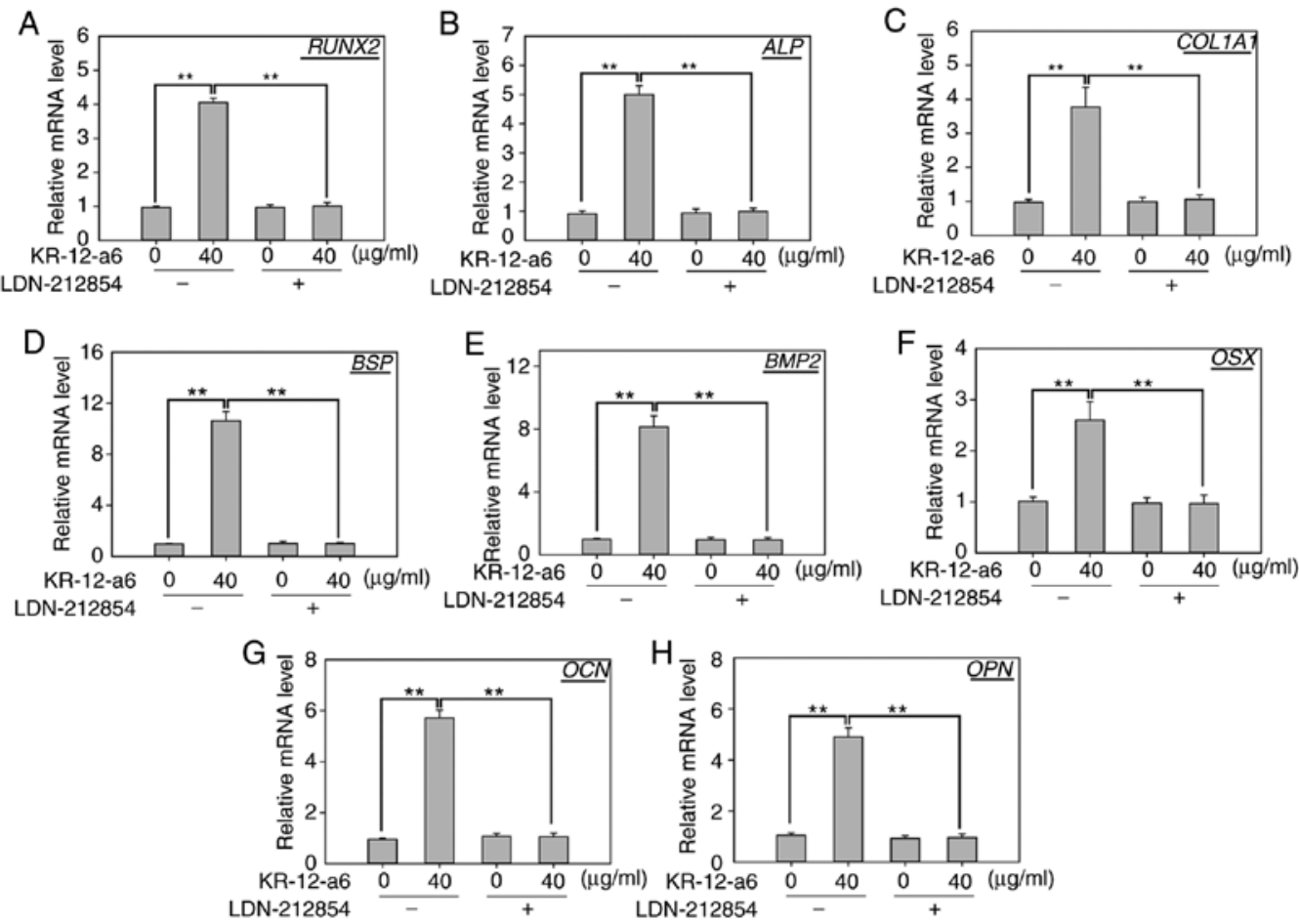

Figure 6. Effects of LDN-212854 on the mRNA expression of osteogenic differentiation-associated genes in KR-12-a6-treated hBMSCs. Reverse transcription-quantitative PCR was performed to examine them RNA expression of (A) RUNX2, (B) $A L P$, (C) COL1A1, (D) BSP, (E) BMP2, (F) $O S X$, (G) $O C N$ and (H) $O P N$ in hBMSCs on day 7 following the various treatments: Control, KR-12-a6, LDN-212854, and KR-12-a6+LDN-212854. Data are presented as the mean $\pm \mathrm{SD}(\mathrm{n}=4) .{ }^{* *} \mathrm{P}<0.01$ vs. KR-12-a6 at $40 \mu \mathrm{g} / \mathrm{ml}$ without $\mathrm{LDN}-212854$. $A L P$, alkaline phosphatase; $B M P$, bone morphogenic protein; $B S P$, bone sialoprotein; COL1A1, type 1 collagen alpha 1 chain; hBMSC, human bone marrow mesenchymal stem cell; $O C N$, osteocalcin; OPN, osteopontin; $O S X$, osterix; $R U N X 2$, runt-related transcription factor 2 .

results in Fig. 4. However, the use of LDN-212854 significantly reduced Smad1/5 phosphorylation in KR-12-a6-treated hBMSCs (Fig. 5). RT-qPCR analysis was then performed to further examine the effects of LDN-212854 (Fig. 6). The results showed that LDN-212854, which inhibited BMP/SMAD signaling, significantly suppressed the mRNA expression of several osteoblastic differentiation-associated genes, including RUNX2 (Fig. 6A), ALP (Fig. 6B), COL1Al (Fig. 6C), BSP (Fig. 6D), BMP2 (Fig. 6E), OSX (Fig. 6F), OCN (Fig. 6G), and $O P N$ (Fig. $6 \mathrm{H}$ ) in hBMSCs at day 7 post-KR-12-a6 treatment. Collectively, these results indicated that BMP/SMAD signaling exerts a positive role in KR-12-a6-induced hBMSC osteogenesis.

\section{Discussion}

Bone infection and osteolysis are common clinical symptoms of osteomyelitis, which requires local or systemic treatment with antibiotics (32). With the rise of drug-resistant bacteria in clinical bone infection, traditional antibiotics have become less effective (18-20). Due to their low drug resistance and excellent antimicrobial properties, AMPs have received increasing attention. AMPs are an important part of the innate immune system, and are regarded as potential substitutes for traditional antibiotics (33). Antimicrobial peptide LL-37 is an important antimicrobial substance that is naturally synthesized in the human body $(7,13)$. It is the first line of defense against local infection and systemic pathogen invasion; more importantly, it does not lead to bacterial resistance (9). However, because of its long amino acid sequence, LL-37 is difficult to develop as a clinical drug for infectious diseases $(10,11)$. As the shortest active fragment of LL-37, KR-12 has the advantages of low cost for synthesis and low cytotoxicity; it is thus predicted to serve an important role in the treatment of infections caused by drug-resistant bacteria $(10,11)$. As an analogue of KR-12, KR-12-a6 has the same antibacterial properties, and is also a potential drug for the clinical treatment of osteomyelitis (15).

Antibiotics that are commonly used to control infection do not promote the formation of new bones; rather, they inhibit the local osteogenic microenvironment and show no effect on the clinical treatment of infection-associated osteolysis $(19,20)$. Studies have shown that antibiotics, including gentamicin and vancomycin, inhibit osteoblast proliferation and differentiation $(19,20)$. In contrast, certain small peptides can control infection while promoting new bone formation (21). Various small AMPs and their analogues may have the ability to promote bone differentiation while also possessing antibacterial properties. Therefore, treating osteomyelitis with AMPs may promote bone repair following infection-induced osteolysis while also controlling the infection. Due to the ability to differentiate into osteoblasts, hBMSCs have been widely used in the study of osteogenic differentiation (24). To verify whether KR-12-a6, a KR-12 analogue, can also affect the osteogenic differentiation of hBMSCs, primary hBMSCs were selected as experimental subjects in this study.

The results showed that KR-12-a6 enhanced the osteogenic differentiation and mineralization of hBMSCs in a dose-dependent manner. KR-12-a6 at $40 \mu \mathrm{g} / \mathrm{ml}$ significantly promoted 
osteogenic differentiation, indicating that KR-12-a6can effectively promote osteogenic differentiation at high concentrations. To accurately evaluate the expression levels of osteoblast-associated genes after KR-12-a6 stimulation, several osteoblast-associated genes were detected at different stages of osteogenic differentiation. During the first 7 days of osteogenesis, the mRNA levels of RUNX2, ALP,COL1A1, BSP and $B M P 2$ increased significantly. In contrast, the late-stage markers of osteogenesis, including $O S X, O C N$ and $O P N$, were upregulated during the second week of osteogenic differentiation. RT-qPCR analysis at different stages revealed that KR-12-a6 enhanced the expression of osteoblast-associated genes in hBMSCs; these effects were more notable at high concentrations of KR-12-a6. These findings suggested that KR-12-a6 may promote the osteogenic differentiation of hBMSCs in vitro. Further mechanistic studies revealed that KR-12-a6 significantly promoted the phosphorylation of Smad1/5. When LDN-212854, a selective BMP inhibitor, was used to block BMP/SMAD signaling, the activation of $\mathrm{p}-\mathrm{Smad} 1 / 5$ was suppressed. Furthermore, the mRNA expression of several osteogenic differentiation-associated genes was also inhibited in KR-12-a6-treated hBMSCs, suggesting the involvement of BMP/SMAD signaling in the KR-12-a6-induced osteogenic differentiation of hBMSCs. However, the current study showed certain limitations. First, results obtained with the use of primary hBMSCs could not fully represent the biophysiological events that happen in vivo and the translational relevance of the study should be strictly confirmed in animal studies in vivo. Second, the activation of BMP/SMAD signaling might not be the only mechanism through which KR-12-a6 enhanced osteogenesis, other potential signaling pathways involved in osteogenesis should also be investigated.

In conclusion, KR-12-a6 promoted the osteogenic differentiation of hBMSCs in a dose-dependent manner, and BMP/SMAD signaling was involved in the process. In addition, considering the potential for the use of AMPs in the treatment of osteomyelitis and other infections, discovering more peptides with antibacterial properties and bone formation regulation is of clear importance for clinical treatment. KR-12-a6 may be an effective drug for the prevention and treatment of local osteomyelitis and infectious osteolysis.

\section{Acknowledgements}

The authors would like to thank Dr Yan C. Cheng (senior scientist, Center for Biomedical Research, the Rockefeller University, United States) for his valuable opinions in the discussion of the present study.

\section{Funding}

This work was supported by the Shaanxi Province Key Research \& Development Projects (grant no. 2017kw-043) and the Talent Support Program of Air Force Military Medical University 'Project Ling Yun' (grant no. cyjhsll).

\section{Availability of data and materials}

The datasets used and/or analyzed during the current study are available from the corresponding author on reasonable request.

\section{Authors' contributions}

LS and LF designed the research and wrote the manuscript; LF and PJ performed experiments and analyzed data; YH and HL collected and analyzed data; LS and LF revised the manuscript and approved the final submission. All authors discussed the results and reviewed the manuscript.

\section{Ethics approval and consent to participate}

The present study was approved by the Ethics Committee of Huazhong University of Science and Technology. Informed consents were obtained from all donors.

\section{Patient consent for publication}

Not applicable.

\section{Competing interests}

The authors declare that they have no competing interests.

\section{References}

1. Bechinger B and Gorr SU: Antimicrobial peptides: Mechanisms of action and resistance. J Dent Res 96: 254-260, 2017.

2. Larrick JW, Hirata M, Balint RF, Lee J, Zhong J and Wright SC: Human CAP18: A novel antimicrobial lipopolysaccharide-binding protein. Infect Immun 63: 1291-1297, 1995.

3. Cowland JB Johnsen AH and Borregaard N: hCAP-18, a cathelin/pro-bactenecin-like protein of human neutrophil specific granules. FEBS Lett 368: 173-176, 1995.

4. Chakraborty K, Ghosh S, Koley H, Mukhopadhyay AK, Ramamurthy T, Saha DR, Mukhopadhyay D, Roychowdhury S, Hamabata T, Takeda Y and Das S: Bacterial exotoxins downregulate cathelicidin (hCAP-18/LL-37) and human beta-defensin 1 (HBD-1) expression in the intestinal epithelial cells. Cell Microbiol 10: 2520-2537, 2008.

5. Agier J, Brzezińska-Błaszczyk E, Żelechowska P, Wiktorska M, Pietrzak J and Różalska S: Cathelicidin LL-37 affects surface and intracellular toll-like receptor expression in tissue mast cells. J Immunol Res 2018: 7357162, 2018.

6. Chamilos G, Gregorio J, Meller S, Lande R, Kontoyiannis DP, Modlin RL and Gilliet M: Cytosolic sensing of extracellular self-DNA transported into monocytes by the antimicrobial peptide LL37. Blood 120: 3699-2707, 2012.

7. Agerberth B, Charo J, Werr J, Olsson B, Idali F, Lindbom L, Kiessling R, Jörnvall $\mathrm{H}$, Wigzell $\mathrm{H}$ and Gudmundsson $\mathrm{GH}$ : The human antimicrobial and chemotactic peptides LL-37 and alpha-defensins are expressed by specific lymphocyte and monocyte populations. Blood 96: 3086-3093, 2000.

8. Dorschner RA, Pestonjamasp VK, Tamakuwala S, Ohtake T, Rudisill J, Nizet V, Agerberth B, Gudmundsson GH and Gallo RL: Cutaneous injury induces the release of cathelicidin anti-microbial peptides active against group A Streptococcus. J Invest Dermatol 117: 91-97, 2001.

9. Duplantier AJ and van Hoek ML: The human cathelicidin antimicrobial peptide LL-37 as a potential treatment for polymicrobial infected wounds. Front Immunol 4: 143, 2013.

10. Wang G: Structures of human host defense cathelicidin LL-37 and its smallest antimicrobial peptide KR-12 in lipid micelles. J Biol Chem 283: 32637-32643, 2008.

11. Mishra B, Epand RF, Epand RM and Wang G: Structural location determines functional roles of the basic amino acids of KR-12, the smallest antimicrobial peptide from human cathelicidin LL-37. RSC Adv: Nov 14, 2013 (Epub ahead of print). doi: $10.1039 /$ C3RA42599A.

12. Rico-Mata R, De Leon-Rodriguez LM and Avila EE: Effect of antimicrobial peptides derived from human cathelicidin LL-37 on Entamoeba histolytica trophozoites. Exp Parasitol 133: 300-306, 2013. 
13. Feng X, Sambanthamoorthy K, Palys T and Paranavitana C: The human antimicrobial peptide LL-37 and its fragments possess both antimicrobial and antibiofilm activities against multidrug-resistant Acinetobacter baumannii. Peptides 49: $131-137,2013$.

14. Luo Y, McLean DT, Linden GJ, McAuley DF, McMullan R and Lundy FT: The naturally occurring host defense peptide, LL-37, and its truncated mimetics KE-18 and KR-12 have selected biocidal and antibiofilm activities against Candida albicans, Staphylococcus aureus, and Escherichia coli in vitro. Front Microbiol 8: 544, 2017.

15. Jacob B, Park IS, Bang JK and Shin SY: Short KR-12 analogs designed from human cathelicidin LL-37 possessing both antimicrobial and antiendotoxic activities without mammalian cell toxicity. J Pept Sci 19: 700-707, 2013.

16. Geurts J, Hohnen A, Vranken T and Moh P: Treatment strategies for chronic osteomyelitis in low- and middle-income countries: Systematic review. Trop Med Int Health 22: 1054-1062, 2017.

17. Mortazavi MM,Khan MA, Quadri SA,Suriya SS, Fahimdanesh KM, Fard SA, Hassanzadeh T, Taqi MA, Grossman H and Tubbs RS Cranial osteomyelitis: A comprehensive review of modern therapies. World Neurosurg 111: 142-153, 2018.

18. Fily F, Ronat JB, Malou N, Kanapathipillai R, Seguin C, Hussein N, Fakhri RM and Langendorf C: Post-traumatic osteomyelitis in Middle East war-wounded civilians: Resistance to first-line antibiotics in selected bacteria over the decade 2006-2016. BMC Infect Dis 19: 103, 2019.

19. Ince A, Schütze N, Karl N, Löhr JF and Eulert J: Gentamicin negatively influenced osteogenic function in vitro. Int Orthop 31 223-228, 2007

20. Mantripragada VP and Jayasuriya AC: Effect of dual delivery of antibiotics (vancomycin and cefazolin) and BMP-7 from chitosan microparticles on Staphylococcus epidermidis and pre-osteoblasts in vitro. Mater Sci Eng C Mater Biol Appl 67: 409-417, 2016

21. Choe H, Narayanan AS, Gandhi DA, Weinberg A, Marcus RE, Lee Z, Bonomo RA and Greenfield EM: Immunomodulatory peptide IDR-1018 decreases implant infection and preserves osseointegration. Clin Orthop Relat Res 473: 2898-2907, 2015.

22. Zou W, Greenblatt MB, Brady N, Lotinun S, Zhai B, de Rivera $H$ Singh A, Sun J, Gygi SP, Baron R, et al: The microtubule-associated protein DCAMKL1 regulates osteoblast function via repression of Runx2. J Exp Med 210: 1793-1806, 2013.

23. Mansour A, Abou-Ezzi G, Sitnicka E, Jacobsen SE, Wakkach A and Blin-Wakkach C: Osteoclasts promote the formation of hematopoietic stem cell niches in the bone marrow. J Exp Med 209: 537-549, 2012.
24. Crane JL and Cao X: Bone marrow mesenchymal stem cells and TGF- $\beta$ signaling in bone remodeling. J Clin Invest 124: 466-472, 2014.

25. Li B: MicroRNA regulation in osteogenic and adipogenic differentiation of bone mesenchymal stem cells and its application in bone regeneration. Curr Stem Cell Res Ther 13: 26-30, 2018.

26. Bidwell JP, Alvarez MB, Hood M Jr and Childress P: Functional impairment of bone formation in the pathogenesis of osteoporosis: The bone marrow regenerative competence. Curr Osteoporos Rep 11: 117-125, 2013.

27. Shen GS, Zhou HB, Zhang H, Chen B, Liu ZP, Yuan Y, Zhou XZ and $\mathrm{Xu} \mathrm{YJ}$ : The GDF11-FTO-PPAR $\gamma$ axis controls the shift of osteoporotic MSC fate to adipocyte and inhibits bone formation during osteoporosis. Biochim Biophys Acta Mol Basis Dis 1864: 3644-3654, 2018.

28. Pavone V, Testa G, Giardina SMC, Vescio A, Restivo DA and Sessa G: Pharmacological therapy of osteoporosis: A systematic current review of literature. Front Pharmacol 8: 803, 2017.

29. Faber C, Stallmann HP, Lyaruu DM, Joosten U, von Eiff C, van NieuwAmerongen A and Wuisman PI: Comparable efficacies of the antimicrobial peptide human lactoferrin 1-11 and gentamicin in a chronic methicillin-resistant Staphylococcus aureus osteomyelitis model. Antimicrob Agents Chemother 49: 2438-2444, 2005.

30. Pittenger MF, Mackay AM, Beck SC, Jaiswal RK, Douglas R, Mosca JD, Moorman MA, Simonetti DW, Craig S and Marshak DR: Multilineage potential of adult human mesenchymal stem cells. Science 284: 143-147, 1999.

31. Livak KJ and Schmittgen TD: Analysis of relative gene expression data using real-time quantitative PCR and the 2(-Delta Delta C(T)) method. Methods 25: 402-408, 2001.

32. Xing K, Huang G, Hua S, Xu G and Li M: Systematic review of randomized controlled trials on antibiotic treatment for osteomyelitis in diabetes. Diabet Med 36: 546-556, 2019.

33. Sierra JM, Fusté E, Rabanal F, Vinuesa T and Viñas M: An overview of antimicrobial peptides and the latest advances in their development. Expert Opin Biol Ther 17: 663-676, 2017.

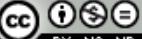

This work is licensed under a Creative Commons Attribution-NonCommercial-NoDerivatives 4.0 International (CC BY-NC-ND 4.0) License. 